\title{
A High-Order Discontinuous Galerkin Solver for Multiphase Flows
}

\author{
Juan Manzanero, Carlos Redondo, Gonzalo Rubio, Esteban Ferrer, \\ Eusebio Valero, Susana Gómez-Álvarez, and Ángel Rivero-Jiménez
}

\section{Introduction}

Multiphase flow is not a canonical problem, therefore different models can be found in the literature. Volume Of Fluid (VOF) model [9] is amongst the simplest. It defines a single set of momentum equations shared by all phases, whilst the volume fraction (fraction of a particular infinitesimal control volume which is occupied by each phase) is tracked throughout the domain following an advection equation. Phase-field methods [11] conserve the simplicity of VOF whilst increasing the physical meaning of the evolution equation of the fluids present in the simulation. The volume fraction is substituted by a phase-field parameter, which identifies each phase. In this work, the Cahn-Hilliard equation [4] is chosen to model the evolution of the phase-field parameter.

The introduced model is discretised in space using a high-order discontinuous Galerkin method. These methods have been gaining popularity for the discretisation of conservation laws, such as the Navier-Stokes equations [5-7, 13, 16, 22, 26]. Specifically, we use a Discontinuous Galerkin Spectral Element Method (DGSEM) [2] that allows the generation of provably stable schemes [8]. These schemes provide enhanced robustness when compared to classical high-order methods [17-20]. As far as the temporal discretisation is concerned, we use an efficient implicit-

J. Manzanero $(\varangle) \cdot$ C. Redondo $\cdot$ G. Rubio $\cdot$ E. Ferrer $\cdot$ E. Valero

ETSIAE-UPM - School of Aeronautics, Universidad Politécnica de Madrid, Madrid, Spain

Center for Computational Simulation, Universidad Politécnica de Madrid, Boadilla del Monte, Madrid, Spain

e-mail: juan.manzanero@upm.es

S. Gómez-Álvarez · Á. Rivero-Jiménez

Repsol Technology Lab, Móstoles, Madrid, Spain

(C) The Author(s) 2020 
explicit approach that permits maintaining the time step restriction of a typical one phase Navier-Stokes solver. It should be noticed that similar approaches to model multiphase flows have been proposed in the past, see for example [29], where an algorithm to model $\mathrm{N}$ immiscible incompressible fluids with high-order methods is described. However, according to the authors knowledge, this is the first implementation using the DGSEM.

The rest of the paper is organised as follows: in Sect. 2 the governing equations of the model are described. In Sect. 3 the numerical techniques to discretise the described model are introduced. Finally, in Sect. 4 the results of two validation test cases are shown.

\section{Governing Equations}

In this work we model multiphase flows with a phase field approach. The flow field is modelled by means of the incompressible Navier-Stokes equations. The evolution of each of the fluids is modelled with the Cahn-Hilliard equation, which defines a phase field variable, $\phi \in[-1,1]$, that identifies spatial coordinates occupied by fluid $1, \phi=-1$, fluid $2, \phi=1$, or an interface $\phi \in(-1,1)$. The value of the thermodynamic properties of the fluids at each spatial coordinate can be computed as:

$$
\rho(\phi)=\rho_{1}\left(\frac{1-\phi}{2}\right)+\rho_{2}\left(\frac{1+\phi}{2}\right), \eta(\phi)=\eta_{1}\left(\frac{1-\phi}{2}\right)+\eta_{2}\left(\frac{1+\phi}{2}\right),
$$

where $\rho_{i}$ is the density of fluid $i$ whilst $\eta_{i}$ is the dynamic viscosity of fluid $i$. The complete system is built considering first the momentum equation,

$$
\frac{\partial(\rho \mathbf{v})}{\partial t}+\nabla \cdot(\rho \mathbf{v v})=-\nabla p+\frac{1}{R e} \nabla \cdot\left(\eta\left(\nabla \mathbf{v}+\nabla \mathbf{v}^{T}\right)\right)+\frac{3}{\sqrt{2} \varepsilon \operatorname{ReC} a} \mu \nabla \phi+\frac{1}{F r^{2}} \rho \mathbf{e}_{g},
$$

with velocity $\mathbf{v}$, static pressure $p$, Reynolds number $R e=\frac{\rho_{1} u_{0} L}{\eta_{1}}$ (where $u_{o}$ is a reference velocity whilst $L$ is a reference length), Capillary number $C a=\frac{\eta_{1} u_{0}}{\sigma}$ (where $\sigma$ represents the surface tension), Froude number $F r=\frac{u_{0}}{\sqrt{g L}}$, (where $g$ is the gravity acceleration) and $\mathbf{e}_{g}$ is the gravity direction. Second, an artificial compressibility method [25] is used to couple the divergence-free condition,

$$
\frac{\partial p}{\partial t}+\frac{\rho_{0}}{\rho_{1}} \frac{1}{M_{0}^{2}} \nabla \cdot \mathbf{v}=0
$$


where $\rho_{0}=\max \left(\rho_{1}, \rho_{2}\right)$ is a reference density, and $M_{0}$ is the artificial compressibility Mach number. Third, the Cahn-Hilliard equation for the phase field,

$$
\frac{\partial \phi}{\partial t}+\nabla \cdot(\phi \mathbf{v})=M \nabla^{2} \mu, \quad \mu=-\phi+\phi^{3}-\varepsilon^{2} \nabla^{2} \phi,
$$

with $M$ the mobility, and $\varepsilon$ the interface width, the two free parameters of the model. In (2) and (4), $\mu$ represents the chemical potential. Moreover, this equation is designed to minimize the free-energy functional [4], $\mathcal{F}$,

$$
\mathcal{F}(\phi, \nabla \phi)=\int_{\Omega}\left(\frac{1}{4}(1-\phi)^{2}(1+\phi)^{2}+\frac{1}{2} \varepsilon^{2}|\nabla \phi|^{2}\right) d x .
$$

Note that the set of Eqs. (2)-(4) is written in non-dimensional form, where the thermodynamic variables of fluid 1 are taken as reference values, e.g.,

$$
\rho(\phi)=\left(\frac{1-\phi}{2}\right)+\frac{\rho_{2}}{\rho_{1}}\left(\frac{1+\phi}{2}\right), \eta(\phi)=\left(\frac{1-\phi}{2}\right)+\frac{\eta_{2}}{\eta_{1}}\left(\frac{1+\phi}{2}\right) .
$$

The set (2)-(4) can be written as an advection-diffusion system:

$$
\frac{\partial u}{\partial t}+\nabla \cdot \mathbf{F}(u)=\nabla \cdot \mathbf{F}_{v}(u, \mathbf{g})+S(u, \mathbf{g}),
$$

where $u=(\phi, \rho \mathbf{v}, p)$ is the state vector, $\mathbf{g}=\left(\mathbf{g}_{\phi}, \mathbf{g}_{v}, \mathbf{g}_{\mu}\right)=(\nabla \phi, \nabla \mathbf{v}, \nabla \mu)$ is the gradients vector, $\mathbf{F}(u)$ and $\mathbf{F}_{v}(u, \mathbf{g})$ are the inviscid and viscous fluxes respectively, and $S(u, \mathbf{g})$ is a source term,

$$
\begin{gathered}
\mathbf{F}(u)=\left[\begin{array}{c}
\phi \mathbf{v} \\
\rho \mathbf{v} \mathbf{v}+p I_{3} \\
\frac{\rho_{0}}{\rho_{1}} \frac{1}{M_{0}^{2}} \mathbf{v}
\end{array}\right], \mathbf{F}_{v}(u, \mathbf{g})=\left[\begin{array}{c}
\mathbf{g}_{\mu} \\
\eta\left(\mathbf{g}_{v}+\mathbf{g}_{v}^{T}\right) \\
0
\end{array}\right], \\
S(u, \mathbf{g})=\left[\begin{array}{c}
3 \\
\frac{3}{\sqrt{2} \varepsilon \operatorname{ReCa}} \mu \mathbf{g}_{\phi}+\frac{1}{F r^{2}} \rho \mathbf{e}_{g} \\
0
\end{array}\right] .
\end{gathered}
$$

\section{Numerical Methods}

The numerical implementation of (2)-(4) is performed using a high-order discontinuous Galerkin scheme for the spatial discretisation (DGSEM variant) and an implicit-explicit Euler scheme for the time discretisation. 


\subsection{Spatial Discretisation Using a Nodal Discontinuous Galerkin Scheme (DGSEM)}

Discontinuous Galerkin (DG) schemes (see [15]) are constructed by tessellating the domain in non-overlapping elements, where the solution is approximated using polynomials of an arbitrary order, $N$. In this particular implementation, we use a nodal variant of the DG method, and we restrict ourselves to hexahedral elements.

In each element we approximate the solution using polynomials written in a set of local spatial coordinates $\xi=(\xi, \eta, \zeta) \in[-1,1]^{3}$, which are related to the physical space by a transfinite mapping,

$$
\mathbf{x}=(x, y, z)=\mathbf{X}(\boldsymbol{\xi})=\mathbf{X}(\xi, \eta, \zeta)
$$

Using the local coordinates, we write the solution using tensor product Lagrange polynomials,

$$
\left.u(\mathbf{x})\right|_{E} \approx U(\boldsymbol{\xi})=\sum_{i, j, k=0}^{N} U^{i j k}(t) l_{i}(\xi) l_{j}(\eta) l_{k}(\zeta)
$$

where the time-dependent coefficients $U^{i j k}(t)$ are the nodal values of the solution $U$, and $l_{j}(\xi)$ are the Lagrange polynomials based on a set of Gauss points $\left\{\xi_{j}\right\}_{j=0}^{N}$. To handle curvilinear geometries, we use a mapping $\mathbf{X}$ that transforms local and physical spaces. With this mapping, we can construct covariant $\mathbf{a}_{i}$ and contravariant $\mathbf{a}^{i}$ basis, and their associated Jacobian $J$, and metrics matrix $\mathcal{M}$ :

$$
\mathbf{a}_{i}=\frac{\partial \mathbf{X}(\xi)}{\partial \xi_{i}}, \quad \mathbf{a}^{i}=\nabla \xi_{i}=\frac{1}{J} \mathbf{a}_{j} \times \mathbf{a}_{k}, \quad J=\mathbf{a}_{i} \cdot\left(\mathbf{a}_{j} \wedge \mathbf{a}_{k}\right), \quad \mathcal{M}=\left[J \mathbf{a}^{\xi}, J \mathbf{a}^{\eta}, J \mathbf{a}^{\zeta}\right] .
$$

Following [14], we transform the system of Eqs. (7) to local coordinates,

$$
\begin{aligned}
& \frac{\partial}{\partial t}\left\{\begin{array}{c}
J \phi \\
J \rho \mathbf{v} \\
J p
\end{array}\right\}+\nabla_{\xi} \cdot\left\{\begin{array}{c}
\mathcal{M}^{T} \mathbf{v} \phi \\
\mathcal{M}^{T} \rho \mathbf{v}+\mathcal{M}^{T} p \mathcal{I}_{3} \\
\mathcal{M}^{T} \frac{1}{M_{0}^{2}} \mathbf{v}
\end{array}\right\}=\nabla_{\xi} \cdot\left\{\begin{array}{c}
M \mathcal{M}^{T} \mathbf{g}_{\mu} \\
\left.\frac{1}{\operatorname{Re}} \mathcal{M}^{T}\left(\eta\left(\mathbf{g}_{\mathbf{v}}+\mathbf{g}_{\mathbf{v}}^{T}\right)\right)\right\} \\
0
\end{array}\right\} \\
& +J\left\{\begin{array}{c}
0 \\
\frac{1}{F r^{2}} \rho \mathbf{e}_{g}+\frac{3}{\sqrt{2} \operatorname{ReCa\varepsilon }} \mu \mathbf{g}_{\phi} \\
0
\end{array}\right\},
\end{aligned}
$$

with gradients,

$$
J \mathbf{g}_{\mathbf{v}}=\mathcal{M} \nabla_{\xi} \mathbf{v}, \quad J \mathbf{g}_{\phi}=\mathcal{M} \nabla_{\xi} \phi, \quad J \mathbf{g}_{\mu}=\mathcal{M} \nabla_{\xi} \mu
$$


and the chemical potential definition,

$$
J \mu=-J \phi+J \phi^{3}-\varepsilon^{2} \nabla_{\xi} \cdot\left(\mathcal{M}^{T} \mathbf{g}_{\phi}\right) .
$$

We obtain the DG scheme replacing the continuous solution by their polynomial counterpart (10), then multiplying (12), written in compact form (7), by a polynomial test function (with same order $N$ as the solution) $\vartheta$, and we integrate the result in one element $E=[-1,1]^{3}$,

$$
\int_{E} J \vartheta \frac{\partial U}{\partial t}+\int_{E} \vartheta \nabla_{\xi} \cdot \mathbf{F}(U)=\int_{E} \vartheta \nabla_{\xi} \cdot \mathbf{F}_{v}(U, \mathbf{G})+\int_{E} J \vartheta S(U, \mathbf{G}) .
$$

Next, we integrate by parts the terms containing divergences, which yields surface integrals. Since the solution is discontinuous at the inter-element faces, we replace the surface flux by a numerical flux, $\mathbf{F}^{\star}$,

$$
\begin{aligned}
\int_{E} J \vartheta \frac{\partial U}{\partial t} & +\int_{\partial E} \vartheta \mathbf{F}^{\star} \cdot \hat{n} d S-\int_{E} \nabla_{\xi} \vartheta \cdot \mathbf{F}=\int_{\partial E} \vartheta \mathbf{F}_{v}^{\star} \cdot \hat{n} d S \\
& -\int_{E} \nabla_{\xi} \vartheta \cdot \mathbf{F}_{v}+\int_{E} J \vartheta S(U, \mathbf{G}),
\end{aligned}
$$

where $\partial E$ represents the six surfaces of the element E. For the inviscid numerical flux $\mathbf{F}^{\star}$, we use the exact Riemann solver derived in [1], whilst for the viscous numerical flux we use the Symmetric Interior Penalty (SIP) method [27], with the penalty parameter value derived in [24] and recently discussed for the DGSEM in [21]. In (16), $\hat{n}$ is the surface outward normal vector in local coordinates. To obtain the evolution equations for each nodal degree of freedom $U^{i j k}$, we let $\vartheta=l_{i}(\xi) l_{j}(\eta) l_{k}(\zeta)$, and compute the integrals using the Gauss quadrature points (and weights $\left\{w_{i}\right\}$ ) associated to the interpolation points (which provide an accuracy of $2 N+1)$,

$$
\begin{aligned}
J^{i j k} \frac{d U^{i j k}}{d t}+ & \left.\frac{F_{x}^{\star}}{w_{i}}\left(\xi, \eta_{j}, \zeta_{k}\right) l_{i}(\xi)\right|_{\xi=-1} ^{\xi=1}+\left.\frac{F_{y}^{\star}}{w_{j}}\left(\xi_{i}, \eta, \zeta_{k}\right) l_{j}(\eta)\right|_{\eta=-1} ^{\eta=1} \\
& +\left.\frac{F_{z}^{\star}}{w_{k}}\left(\xi_{i}, \eta_{j}, \zeta\right) l_{k}(\zeta)\right|_{\zeta=-1} ^{\zeta=1} \\
& -\sum_{m=0}^{N}\left(\frac{w_{m}}{w_{i}} D_{m i} F_{x}^{m j k}+\frac{w_{m}}{w_{j}} D_{m j} F_{y}^{i m k}+\frac{w_{m}}{w_{k}} D_{m k} F_{z}^{i j m}\right)= \\
& \frac{F_{v, x}^{\star i j k}}{w_{i}}\left(\delta_{i N}-\delta_{i 0}\right)+\frac{F_{v, y}^{\star i j k}}{w_{j}}\left(\delta_{j N}-\delta_{j 0}\right)+\frac{F_{v, z}^{\star i j k}}{w_{k}}\left(\delta_{k N}-\delta_{k 0}\right) \\
& -\sum_{m=0}^{N}\left(\frac{w_{m}}{w_{i}} D_{m i} F_{v, x}^{m j k}+\frac{w_{m}}{w_{j}} D_{m j} F_{v, y}^{i m k}+\frac{w_{m}}{w_{k}} D_{m k} F_{v, z}^{i j m}\right)+J^{i j k} S^{i j k},
\end{aligned}
$$


where $F^{i j k}=F\left(U^{i j k}\right)$ and $F_{v}^{i j k}=F_{v}\left(U^{i j k}, \mathbf{G}^{i j k}\right)$, being $\mathbf{G}^{i j k}$ the nodal values of the gradient $\mathbf{G}$. The symbol $\delta_{i k}$ represents the Kronecker delta. The derivation matrix $D_{i j}$ is defined as $D_{i j}=l_{j}^{\prime}\left(\xi_{i}\right)$. To compute the gradient $\mathbf{G}$, we perform the weak formulation of (13),

$$
\int_{E} J \boldsymbol{\tau} \cdot \mathbf{G}=\int_{\partial E} U^{\star} \mathcal{M}^{T} \cdot \boldsymbol{\tau} d S-\int_{E} U \nabla_{\xi} \cdot\left(\mathcal{M}^{T} \cdot \boldsymbol{\tau}\right),
$$

where $\tau$ is an arbitrary vector test function (from the order $N$ polynomials space). Since we use the SIP method, we use solution averages to couple inter-element fluxes, $U^{\star}=\{\{U\}$. All the integrals involved in (18) are computed discretely similar to those in (16), i.e.,

$$
\begin{aligned}
J^{i j k} \tau_{i j k}^{d} G_{d}^{i j k}=\left.\frac{U^{\star}\left(\xi, \eta_{j}, \zeta_{k}\right) J a_{d}^{\xi}\left(\xi, \eta_{j}, \zeta_{k}\right)}{w_{i}} l_{i}(\xi)\right|_{\xi=-1} ^{\xi=1} \\
+\left.\frac{U^{\star}\left(\xi_{i}, \eta, \zeta_{k}\right) J a_{d}^{\eta}\left(\xi_{i}, \eta, \zeta_{k}\right)}{w_{j}} l_{j}(\eta)\right|_{\eta=-1} ^{\eta=1}+\left.\frac{U^{\star}\left(\xi_{i}, \eta_{j}, \zeta\right) J a_{d}^{\zeta}\left(\xi_{i}, \eta_{j}, \zeta\right)}{w_{k}} l_{k}(\zeta)\right|_{\zeta=-1} ^{\zeta=1} \\
\quad-\sum_{m=0}^{N}\left(\frac{w_{m}}{w_{i}} J a_{d}^{\xi, i j k} D_{m i} U^{m j k}+\frac{w_{m}}{w_{j}} J a_{d}^{\eta, i j k} D_{m j} U^{i m k}+\frac{w_{m}}{w_{k}} J a_{d}^{\zeta, i j k} D_{m k} U^{i j m}\right) .
\end{aligned}
$$

The gradient nodal values $G_{d}^{i j k}$ are introduced in the viscous fluxes $F_{v}\left(U^{i j k}, \mathbf{G}^{i j k}\right)$ of (17) hence completing the discretisation of (16). Note that one needs to compute $\mathbf{g}_{\phi}$ before computing $\mu$ and its gradient $\mathbf{g}_{\mu}$.

\subsection{Time Integration Using IMplicit-EXplicit (IMEX) and Runge-Kutta Schemes}

The time integration of (17) is performed with a combination of forward and backwards Euler and explicit Runge-Kutta schemes. On the one hand, the NavierStokes equations are integrated by means of a third order explicit Runge-Kutta (RK3) scheme [28]. On the other hand, the Cahn-Hilliard equation is integrated with a combination of explicit RK3 for the phase field advection, forward Euler for the chemical free-energy, and backwards Euler for the interfacial energy,

$$
\frac{\phi^{n+1}-\phi^{n}}{\Delta t}+\nabla \cdot(\mathbf{v} \phi)^{R K 3}=\nabla^{2}\left(-\phi^{n}+\left(\phi^{n}\right)^{3}-\varepsilon^{2} \nabla^{2} \phi^{n+1}\right) .
$$

The reason behind this choice, is that the numerical stiffness of the bi-Laplacian $\left(\nabla^{4} \phi\right)$ operator prevents from using an explicit method, as restricts the time-step $\Delta t$ 
to unpractical values. We only treat implicitly the interfacial energy since it yields a constant Jacobian matrix, represented by $J^{\nabla_{2}}$. In particular, the linear system to solve is,

$$
\left[J^{\nabla_{2}}+\frac{\mathcal{I}}{\Delta t}\right] \phi^{n+1}=\frac{\phi^{n}}{\Delta t}-\nabla \cdot(\mathbf{v} \phi)^{R K 3}+\nabla^{2}\left(-\phi^{n}+\left(\phi^{n}\right)^{3}\right) .
$$

The Jacobian matrix is computed numerically (see [3]) and a LU factorisation is performed only at the first time step. In each following iteration, the RHS of (21) is computed and the linear system is solved by means of forward and backward substitutions. Both the LU factorisation and the forward and backward substitutions are performed with the library MKL-PARDISO [23].

\section{Validation}

The proposed methodology is tested with two test cases. First, the validity of the discontinuous Galerkin discretisation of the Cahn-Hilliard equation is tested with a benchmark spinodal decomposition problem [12]. Second, the validity of the coupled Cahn-Hilliard/Navier-Stokes system is tested with a two dimensional rising bubble test [10].

\subsection{Spinodal Decomposition}

This test problem considers an initial mixture of two fluids. These fluids are immiscible, therefore they tend to separate to minimise their free energy (5). As stated before, the geometry, initial condition and fluid parameters are taken from [12]. In particular, the initial condition for this benchmark problem is:

$$
\begin{array}{r}
\phi(x, y)=-0.05\left[\cos (0.105 x) \cos (0.11 y)+[\cos (0.13 x) \cos (0.087 y)]^{2}\right. \\
+\cos (0.025 x-0.15 y) \cos (0.07 x-0.02 y)] .
\end{array}
$$

The physical domain is a "T" shape with a total height of 120 units, a total width of 100 units, and horizontal and vertical section widths of 20 units (Fig. 1). No-flux boundary conditions are applied at the boundaries. Following [12] mobility is set to $M=10$, whilst the interface width is set to $\varepsilon=3.16$. The physical domain is discretised with an unstructured mesh of 326 elements and a polynomial order of $N=4$. For the time discretization, we use a time step $\Delta t=10^{-3}$.

Figure 1 shows qualitatively how the different phases separate, whilst Fig. 2 shows quantitatively the evolution of the total free energy with time. In Fig. 2 

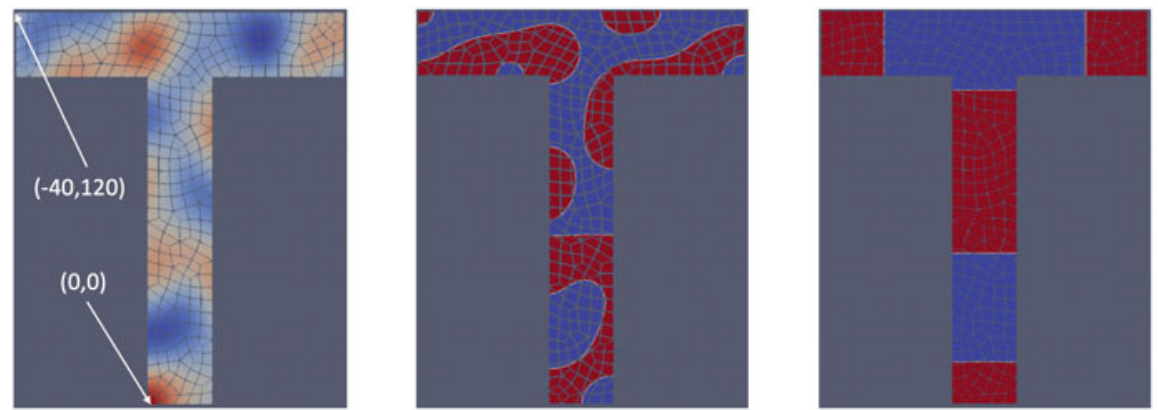

Fig. 1 "T" domain for the spinodal decomposition. Initial condition (left figure) and evolution with time (the right figure is the steady-state solution)

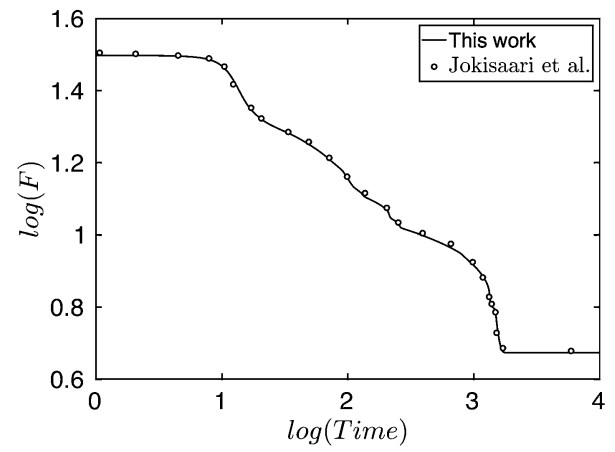

Fig. 2 Evolution of total free energy (5) with time

the results of this work are compared with those obtained in [12], validating the proposed method.

\subsection{Rising Bubble}

This test case considers a bubble of light fluid submerged in a heavy fluid, both subjected to a gravitational field. Following [10] the initial configuration, see Fig. 3, consists of a bubble of radius $r=0.25$ centred at $[0.5,0.5]$ in a $[1 \times 2]$ domain. A no-slip boundary condition is used at the top and the bottom of the domain whilst a free slip condition is enforced at the vertical walls. Following [10], the Reynolds number is set to $R e=35$ whilst $\sigma$ and $\varepsilon$ are set to 24.5 and 0.03125 respectively (this gives a Eötvös number $E o=10$ ) whilst both density and viscosity ratios are set to $\rho_{1} / \rho_{2}=\mu_{1} / \mu_{2}=10$. The gravitational acceleration is $g=0.98$. The problem is discretised with $16 \times 32$ elements with a polynomial order of $N=4$, and a time step $\Delta t=4 \cdot 10^{-6}$. 


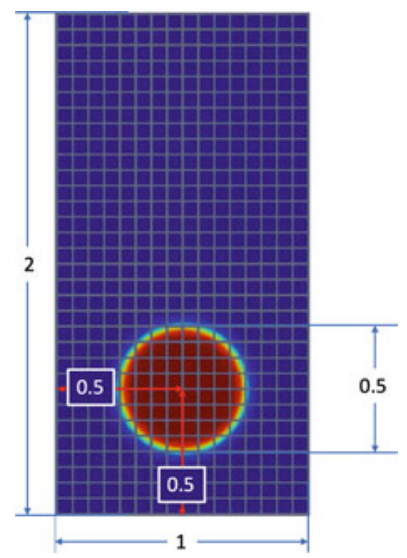

Fig. 3 Initial condition of the rising bubble test problem

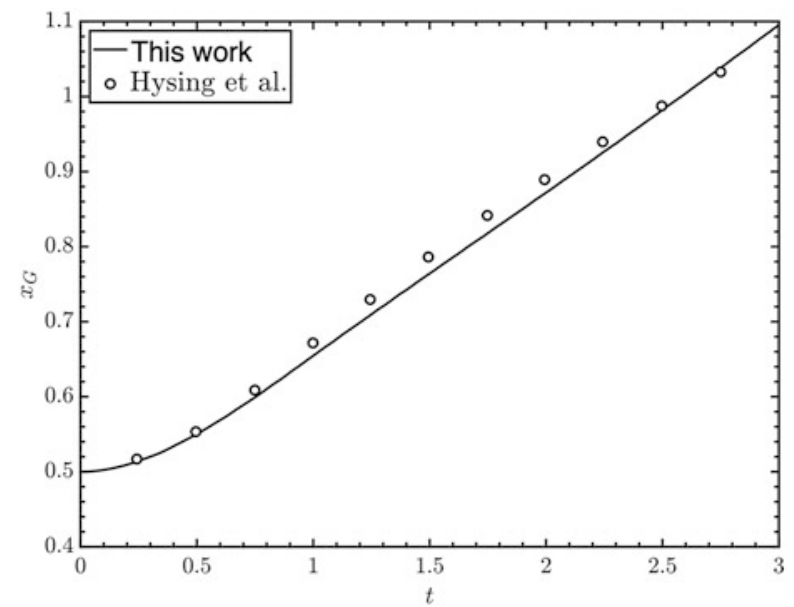

Fig. 4 Evolution of the center of mass of the bubble with time

This test case is quantitively compared with the results of [10] in Fig. 4 with satisfactory results. It should be mentioned that the benchmark results of [10] are obtained with a sharp-interface model which may explain the small disagreement in the evolution of the center of mass shown in Fig. 4.

\section{Conclusions}

A method to model incompressible two phases flows is introduced. The model solves the incompressible Navier-Stokes equations coupled with the Cahn-Hilliard equation to track the evolution of the different fluids. The model is discretised in 
space using a discontinuous Galerkin spectral element method (DGSEM) whilst an efficient implicit-explicit approach is used to advance in time. The validity of the model is shown with two test cases. A spinodal decomposition benchmark problem is solved to validate the Cahn-Hilliard solver whilst a rising-bubble test problem is solved to validate the coupled Cahn-Hilliard-Navier-Stokes system. Both test cases are solved showing good agreement with the literature, and proving the accuracy and robustness of the proposed method.

Acknowledgements This work has been partially supported by REPSOL under the research grant P180021090. This work has been partially supported by Ministerio de Economía y Competitividad under the research grant TRA2015-67679-C2-2-R and under the research grant EUIN2017-88294 (Gonzalo Rubio). The authors acknowledge the computer resources and technical assistance provided by the Centro de Supercomputación y Visualización de Madrid (CeSViMa).

\section{References}

1. Bassi, F., Massa, F., Botti, L., Colombo, A.: Artificial compressibility Godunov fluxes for variable density incompressible flows. Comput. Fluids 169, 186-200 (2018)

2. Black, K.: A conservative spectral element method for the approximation of compressible fluid flow. Kybernetika 35(1), 133-146 (1999)

3. Browne, O.M., Rubio, G., Ferrer, E., Valero, E.: Sensitivity analysis to unsteady perturbations of complex flows: a discrete approach. Int. J. Numer. Methods Fluids 76(12), 1088-1110 (2014)

4. Cahn, J.W., Hilliard, J.E.: Free energy of a nonuniform system. I. Interfacial free energy. J. Chem. Phys. 28(2), 258-267 (1958)

5. Cockburn, B., Shu, C.W.: The local discontinuous Galerkin method for time-dependent convection-diffusion systems. SIAM J. Numer. Anal. 35(6), 2440-2463 (1998)

6. Ferrer, E.: An interior penalty stabilised incompressible discontinuous Galerkin-Fourier solver for implicit large eddy simulations. J. Comput. Phys. 348, 754-775 (2017)

7. Fraysse, F., Redondo, C., Rubio, G., Valero, E.: Upwind methods for the Baer-Nunziato equations and higher-order reconstruction using artificial viscosity. J. Comput. Phys. 326, 805 827 (2016)

8. Gassner, G.J., Winters, A.R., Kopriva, D.A.: Split form nodal discontinuous Galerkin schemes with summation-by-parts property for the compressible Euler equations. J. Comput. Phys. 327, 39-66 (2016)

9. Hirt, C.W., Nichols, B.D.: Volume of fluid (VOF) method for the dynamics of free boundaries. J. Comput. Phys. 39(1), 201-225 (1981)

10. Hysing, S., Turek, S., Kuzmin, D., Parolini, N., Burman, E., Ganesan, S., Tobiska, L.: Quantitative benchmark computations of two-dimensional bubble dynamics. Int. J. Numer. Methods Fluids 60(11), 1259-1288

11. Jacqmin, D.: Calculation of two-phase Navier-Stokes flows using phase-field modeling. J. Comput. Phys. 155(1), 96-127 (1999)

12. Jokisaari, A., Voorhees, P., Guyer, J., Warren, J., Heinonen, O.: Benchmark problems for numerical implementations of phase field models. Comput. Mater. Sci. 126, 139-151 (2017)

13. Kompenhans, M., Rubio, G., Ferrer, E., Valero, E.: Adaptation strategies for high order discontinuous Galerkin methods based on Tau-estimation. J. Comput. Phys. 306, 216-236 (2016)

14. Kopriva, D.A.: Metric identities and the discontinuous spectral element method on curvilinear meshes. J. Sci. Comput. 26(3), 301 (2006) 
15. Kopriva, D.A.: Implementing Spectral Methods for Partial Differential Equations: Algorithms for Scientists and Engineers. Springer, Berlin (2009)

16. Manzanero, J., Ferrer, E., Rubio, G., Valero, E.: Design of a Smagorinsky Spectral Vanishing Viscosity turbulence model for discontinuous Galerkin methods. Comput. Fluids, 104440 (2020)

17. Manzanero, J., Rubio, G., Ferrer, E., Valero, E., Kopriva, D.A.: Insights on aliasing driven instabilities for advection equations with application to Gauss-Lobatto discontinuous Galerkin methods. J. Sci. Comput. 75(3), 1262-1281 (2018)

18. Manzanero, J., Rubio, G., Kopriva, D.A., Ferrer, E., Valero, E.: Entropy-stable discontinuous Galerkin approximation with summation-by-parts property for the incompressible NavierStokes/Cahn-Hilliard system (2019). arXiv:1910.11252.

19. Manzanero, J., Rubio, G., Kopriva, D.A., Ferrer, E., Valero, E.: A free-energy stable nodal discontinuous Galerkin approximation with summation-by-parts property for the CahnHilliard equation. J. Comput. Phys. 403, 109072 (2020)

20. Manzanero, J., Rubio, G., Kopriva, D.A., Ferrer, E., Valero, E.: An entropy-stable discontinuous Galerkin approximation for the incompressible Navier-Stokes equations with variable density and artificial compressibility. J. Comput. Phys. 408, 109241 (2020)

21. Manzanero, J., Rueda-Ramírez, A.M., Rubio, G., Ferrer, E.: The Bassi Rebay 1 scheme is a special case of the symmetric interior penalty formulation for discontinuous Galerkin discretisations with Gauss-Lobatto points. J. Comput. Phys. 363, 1-10 (2018)

22. Rueda-Ramírez, A.M., Manzanero, J., Ferrer, E., Rubio, G., Valero, E.: A p-multigrid strategy with anisotropic p-adaptation based on truncation errors for high-order discontinuous Galerkin methods. J. Comput. Phys. 378, 209-233 (2019)

23. Schenk, O., Gärtner, K.: Solving unsymmetric sparse systems of linear equations with PARDISO. Futur. Gener. Comput. Syst. 20(3), 475-487 (2004)

24. Shahbazi, K.: Short note: an explicit expression for the penalty parameter of the interior penalty method. J. Comput. Phys. 205(2), 401-407 (2005)

25. Shen, J.: Pseudo-compressibility methods for the unsteady incompressible Navier-Stokes equations. In: Proceedings of the 1994 Beijing Symposium on Nonlinear Evolution Equations and Infinite Dynamical Systems, pp. 68-78 (1997)

26. Wang, Z.J., Fidkowski, K., Abgrall, R., Bassi, F., Caraeni, D., Cary, A., Deconinck, H., Hartmann, R., Hillewaert, K., Huynh, H.T., et al.: High-order CFD methods: current status and perspective. Int. J. Numer. Methods Fluids 72(8), 811-845 (2013)

27. Wheeler, M.F.: An elliptic collocation-finite element method with interior penalties. SIAM J. Numer. Anal. 15(1), 152-161 (1978)

28. Williamson, J.: Low-storage Runge-Kutta schemes. J. Comput. Phys. 35(1), 48-56 (1980)

29. Yang, Z., Dong, S.: Multiphase flows of $\mathrm{N}$ immiscible incompressible fluids: an outflow/open boundary condition and algorithm. J. Comput. Phys. 366, 33-70 (2018)

Open Access This chapter is licensed under the terms of the Creative Commons Attribution 4.0 International License (http://creativecommons.org/licenses/by/4.0/), which permits use, sharing, adaptation, distribution and reproduction in any medium or format, as long as you give appropriate credit to the original author(s) and the source, provide a link to the Creative Commons licence and indicate if changes were made.

The images or other third party material in this chapter are included in the chapter's Creative Commons licence, unless indicated otherwise in a credit line to the material. If material is not included in the chapter's Creative Commons licence and your intended use is not permitted by statutory regulation or exceeds the permitted use, you will need to obtain permission directly from the copyright holder. 